\title{
Thyroid Hormone Opposes Some Glucocorticoid Effects on Glycogen Content and Lipid Synthesis in Developing Fetal Rat Lung ${ }^{1}$
}

\author{
SEAMUS A. ROONEY, LAURICE I. GOBRAN, AND ARTHUR J. CHU \\ Division of Perinatal Medicine, Department of Pediatrics, Yale University School of Medicine, \\ New Haven, Connecticut 06510
}

\begin{abstract}
Because of current interest in use of a combination of glucocorticoid and thyroid hormones for prevention of respiratory distress syndrome we examined the effects of dexamethasone and triiodothyronine $\left(T_{3}\right)$, alone and in combination, on glycogen content and rates of fatty acid and phosphatidylcholine synthesis in fetal rat lung. The hormones were administered to the mothers on the 2 days before delivery on days 17-22 of gestation. Both hormones increased the rate of choline incorporation into phosphatidylcholine, an index of surfactant synthesis, on day 20 just prior to the normal developmental surge but had no effect on this parameter on days 19, 21, or 22 . There is a developmental increase in lung glycogen on days 17-20 with a decrease thereafter and a developmental increase in the rate of fatty acid synthesis between days $\mathbf{2 0}$ and 21 . The increases in glycogen content and fatty acid synthesis were accelerated by dexamethasone and prevented by $T_{3}$ and when the hormones were administered together $T_{3}$ antagonized the stimulatory effects of dexamethasone on these parameters. Both dexamethasone and $T_{3}$ accelerated the normal developmental decrease in lung glycogen later in gestation and the effects of the two hormones on this parameter were additive. The combination of dexamethasone and $T_{3}$ led to significantly smaller fetuses and increased mortality late in gestation. These data show that glucocorticoid and thyroid hormones have opposite as well as common effects on parameters of fetal lung maturation. Although the relationship between changes in lung glycogen or fatty acid synthesis and surfactant production are not known the combination of hormones may be beneficial at certain gestational ages but harmful at others. (Pediatr Res 20: 545-550, 1986)
\end{abstract}

\section{Abbreviations}

$T_{3}$, triiodothyronine

RDS, respiratory distress syndrome

Glucocorticoids and thyroid hormone have been shown to accelerate fetal lung maturation and stimulate surfactant production in several animal species by biochemical, physiological, and morphological criteria (1). Glucocorticoids have long been

Received October 24, 1985; accepted February 3, 1986.

Correspondence Seamus A. Rooney, Ph.D., Yale Dept. of Pediatrics, P.O. Box 3333, New Haven, CT 06510.

This work was supported by Grant HD-10192 from the National Institute of Child Health and Human Development. AJC was supported in part by Training Grant HL-07410 from the National Heart, Lung and Blood Institute

${ }^{1}$ Presented in part of the 13th International Congress of Biochemistry, Amsterdam, The Netherlands, August 25-30, 1985. used prophylactically to accelerate fetal lung maturation and prevent RDS in human newborns $(2,3)$ and in at least one clinical study (4) thyroxine was also reported to be effective in this regard. However, the findings in a recent multicenter study that glucocorticoids are not as efficacious in preventing RDS as earlier believed and that its effects may be largely confined to female infants (5) has led to interest in use of a combination of glucocorticoids and thyroid hormone for prevention of RDS (6). Prior to such clinical use, however, it is desirable to have information on the interaction of these hormones in the acceleration of lung maturation in animals.

Previous studies in animals have shown that a combination of glucocorticoid and thyroid hormones is more effective than either hormone alone in accelerating fetal lung maturation and stimulating surfactant production. In a morphological study in the fetal rat, Hitchcock (7) reported maximal acceleration of lung maturation when glucocorticoids and thyroxine were both present. Similarly, a synergistic interaction between glucocorticoids and thyroid hormone in stimulating synthesis of phosphatidylcholine and disaturated phosphatidylcholine, the major component of pulmonary surfactant (1), was demonstrated in fetal rat lung in vivo $(8,9)$ and in fetal rat $(10,11)$, rabbit $(12)$, and human (13) lung in culture.

In addition to morphology and synthesis of surfactant phospholipids, lung maturation may also be assessed by other criteria. An increase followed by a decrease in glycogen content is a characteristic feature of fetal lung development $(1,14)$. Although there is a temporal relationship between the decrease in lung glycogen and appearance of lamellar inclusion bodies (15) and increased phosphatidylcholine synthesis (14) and it has been speculated that glycogen may provide substrate for surfactant synthesis (16), a direct precursor-product relationship has not been established (1). Nevertheless, hormones that accelerate lung maturation and stimulate surfactant production in the fetus also promote glycogen depletion (1). Glucocorticoids decreased lung glycogen in vivo in the fetal rabbit $(17,18)$ and mouse $(19)$ while dexamethasone and thyroxine had a similar effect in cultured fetal rat lung explants (20). The influence of a combination of these two hormones on this parameter of lung maturation, however, has not previously been reported.

A developmental increase in the rate of de novo fatty synthesis and in the activity of acetyl-CoA carboxylase with increasing gestational age was reported in fetal rat lung (21). A similar developmental increase in the activity of fatty-acid synthase was reported in fetal rabbit lung (22). Dexamethasone was reported to enhance fatty acid synthesis in fetal rabbit lung explants (23) while a role for thyroid hormone in regulation of pulmonary fatty acid synthesis was suggested in the fetal rabbit (22) and adult rat (24).

The objectives of this study were, therefore, 3-fold: to examine the effects of glucocorticoid and thyroid hormone, alone and in 
combination, on the glycogen content and rate of de novo fatty acid synthesis in fetal rat lung; to determine to what extent the effects of these hormones were dependent on gestational age; and to determine if there was any relationship between any developmental or hormone-induced changes in these parameters of lung maturation and changes in the rate of phosphatidylcholine synthesis.

\section{MATERIALS AND METHODS}

Animals. Timed pregnant Sprague-Dawley rats (Charles River, Wilmington, MA) were injected intramuscularly with dexamethasone, $\mathrm{T}_{3}$ in $0.9 \% \mathrm{NaCl}$, the two hormones together, or $0.9 \%$ $\mathrm{NaCl}$ (controls) on the 2 days prior to killing on 17-22 days gestation. (The day after mating was considered day 1 ; term is 22 days.) Dexamethasone was administered once in the morning and once in the evening ( $1 \mathrm{mg} / \mathrm{kg}$ each injection) while $\mathrm{T}_{3}$ was injected only in the morning $(7 \mathrm{mg} / \mathrm{kg} / \mathrm{day})$. The hormone doses used were those previously shown to optimally increase the rate of choline incorporation into phosphatidylcholine in fetal rat lung at 20 days gestation (8).

The mothers were killed by decapitation after concussion and the fetuses were delivered by cesarean section. Newborns were delivered spontaneously (on day 22) and kept with the mother for an additional day. These animals were not injected. Fetuses and newborns were killed by decapitation. Lungs from two to three animals in each litter were rapidly excised and immediately frozen in dry ice-acetone for glycogen assay. The remaining fetuses were pooled, the average body weight was obtained, and the lungs were excised and placed in chilled Krebs-Ringer phosphate buffer ( $\mathrm{pH}$ 7.4) for subsequent measurement of fatty acid synthesis or choline incorporation into phosphatidylcholine.

Fatty acid synthesis. Fatty acid synthesis was assessed by measuring the rate of incorporation of ${ }^{3} \mathrm{H}$ from ${ }^{3} \mathrm{H}_{2} \mathrm{O}$ into total lipid fatty acid in lung slices. Use of ${ }^{3} \mathrm{H}_{2} \mathrm{O}$ rather than acetate or other substrate overcomes problems associated with differences in substrate pool size (25). The method was a modification of that described by Maniscalco et al. (21). Slices $(0.5 \mathrm{~mm}$ thick) were prepared from the freshly excised lungs with a McIlwain tissue chopper (Brinkmann, Westbury, NY) and incubated in 2 $\mathrm{ml} \mathrm{Krebs-Ringer} \mathrm{phosphate} \mathrm{buffer} \mathrm{(pH} \mathrm{7.4)} \mathrm{containing} 5 \mathrm{mM}$ glucose in capped $20 \mathrm{ml}$ scintillation vials in a shaking water bath at $37^{\circ} \mathrm{C}$. Lung tissue from each litter was distributed among two to four vials (3-13 $\mathrm{mg}$ protein each). After a 10-min preincubation period ${ }^{3} \mathrm{H}_{2} \mathrm{O}(0.5 \mathrm{mCi})$ was added and the incubation continued. The reaction was stopped after 0 or $2 \mathrm{~h}$ by addition of $7.5 \mathrm{ml}$ chilled $0.9 \% \mathrm{NaCl}$. The tissue was further washed with additional aliquots of saline and finally homogenized in $1.8 \mathrm{ml}$. An aliquot of the homogenate was removed for protein assay by the method of Lowry et al. (26) and a further aliquot $(1.6 \mathrm{ml})$ for lipid extraction with chloroform and methanol by the method of Bligh and Dyer (27). The total lipids were evaporated to dryness under $\mathrm{N}_{2}, 2 \mathrm{ml} 14 \%$ boron trifluoride in methanol (Sigma, St Louis, MO) were added, and the mixture was incubated at $85^{\circ} \mathrm{C}$ for $1 \mathrm{~h}$. On cooling to room temperature, $1 \mathrm{ml}$ saturated $\mathrm{NaCl}$ was added and the fatty acid methyl esters were extracted with two 4-ml aliquots of hexane and purified by thinlayer chromatography on LK5D silica gel plates (Whatman, Clifton, NJ) in hexane:diethyl ether:acetic acid $(80: 20: 1, \mathrm{v} / \mathrm{v} / \mathrm{v}$. The fatty acid methyl ester band was detected by exposure to iodine vapor and identified by comparison with standards. Radioactivity was measured directly on the gel by liquid scintillation counting in Aquasol (New England Nuclear, Boston, MA) containing $3 \%$ water. The counting efficiency was $45 \%$. The amount of radioactivity in the incubation medium was also measured to obtain the initial specific activity. Under these conditions the rate of ${ }^{3} \mathrm{H}$ incorporation into fatty acid was linear with time for at least $3 \mathrm{~h}$.

Rate of choline incorporation into phosphatidylcholine. The rate of [methyl- $\left.{ }^{14} \mathrm{C}\right]$ choline incorporation into phosphatidylcho- line in lung slices was measured in Krebs-Ringer phosphate buffer as described previously $(17,19)$. Because of possible differences in the intracellular pool sizes of choline or phosphorylated choline intermediates it is possible that this measurement is not a true reflection of phosphatidylcholine synthesis. Nevertheless in developmental and hormonal studies on the fetal lung the rate of choline incorporation into phosphatidylcholine in slices has shown good correlation with increased mass of phosphatidylcholine and with increased cholinephosphate cytidylyltransferase activity $(1,14)$.

Glycogen assay. Glycogen was assayed as described previously (28). In this procedure glycogen breakdown is coupled to generation of NADPH which is measured fluorometrically (29). The rapidly frozen lungs were stored at $-70^{\circ} \mathrm{C}$ until assayed.

Chemicals. Radiochemicals were from New England Nuclear, dexamethasone sodium phosphate $(4 \mathrm{mg} / \mathrm{ml})$ from Elkins-Sinn (Cherry Hill, NJ) or Merck, Sharp \& Dohme (West Point, PA) and $\mathrm{T}_{3}$ and other biochemicals from Sigma (St. Louis, MO). Other chemicals were reagent grade or better.

Statistics. Each glycogen value is from a single fetus or newborn (two to three per litter) assayed in duplicate while each value from the fatty acid and choline incorporation experiments is the mean of two to three determinations from a single litter. Groups were compared by one way analysis of variance with Student-Newman-Keuls test; $p<0.05$ was considered statistically significant.

\section{RESULTS}

Developmental changes in glycogen content and in rates of fatty acid synthesis and choline incorporation into phosphatidylcholine in fetal and newborn rat lung are shown in Figure 1. There was an increase in lung glycogen up to day 20, when the highest level was reached, followed by a decrease. The rate of fatty acid synthesis changed little between 19 and 20 days, then increased more than $50 \%$ to reach a plateau at $21-22$ days and decreased to less than the 19- to 20-day rate in the newborn period. The rate of choline incorporation into phosphatidylcholine more than tripled between 19-20 days gestation and 1 day after birth. These profiles are similar to those previously reported for glycogen content $(28,30-32)$ and phosphatidylcholine synthesis $(28,33-36)$ in the rat. Similar developmental profiles have been reported in other species $(1,14)$. In one previous study $(21)$ a developmental profile for rat lung de novo fatty acid synthesis similar to that in Figure 1 was also reported.

The effects of dexamethasone and $\mathrm{T}_{3}$, alone and in combination, on fatty acid synthesis in developing rat lung are shown in Table 1. The rate of fatty acid synthesis was significantly increased by dexamethasone on day 20 but unaffected by this hormone at the other ages examined. On days 21 and 22 fatty acid synthesis was significantly lower in the $T_{3}$-treated group than in the controls. Thus the developmental increase in fatty acid synthesis which occurs normally on day 21 was accelerated by the glucocorticoid and prevented by the thyroid hormone. Fatty acid synthesis in the combined hormone-treated group was significantly different from that in both the control and dexamethasone-treated groups on days 20 and 21 . Thus $\mathrm{T}_{3}$ antagonized both the normal developmental increase in fatty acid synthesis and that induced by dexamethasone.

The effects of the hormones on the glycogen content of the fetal lung are shown in Table 2. Compared to the controls, dexamethasone increased lung glycogen on days 17-19 and decreased it on days 21-22. Thus dexamethasone increased lung glycogen at the period in gestation when there is a normal developmental increase in this parameter and decreased it when it is normally decreasing (Fig. 1). Since the peak in lung glycogen occurred on day 19 in the glucocorticoid-treated group but on day 20 in the controls (Fig. 1), the hormone accelerated lung maturation by one day as determined by this parameter. $T_{3}$, on the other hand, had no effect on lung glycogen on days 17 and 
18 but decreased it on days 19-22. This hormone, therefore, prevented the normal developmental increase in lung glycogen between days 18 and 20, although not between days 17 and 18, and accelerated the developmental decrease which normally occurs after day 20 (Fig. 1). When compared to controls, the two hormones together increased lung glycogen on days 17 and 18 and decreased it on days 19-22. However, the increase on days 17 and 18 was less than that produced by dexamethasone alone suggesting that this effect of the glucocorticoid was also antagonized by $T_{3}$. The increase produced by dexamethasone alone on day 19 was reversed by $T_{3}$ as there was less lung glycogen in the

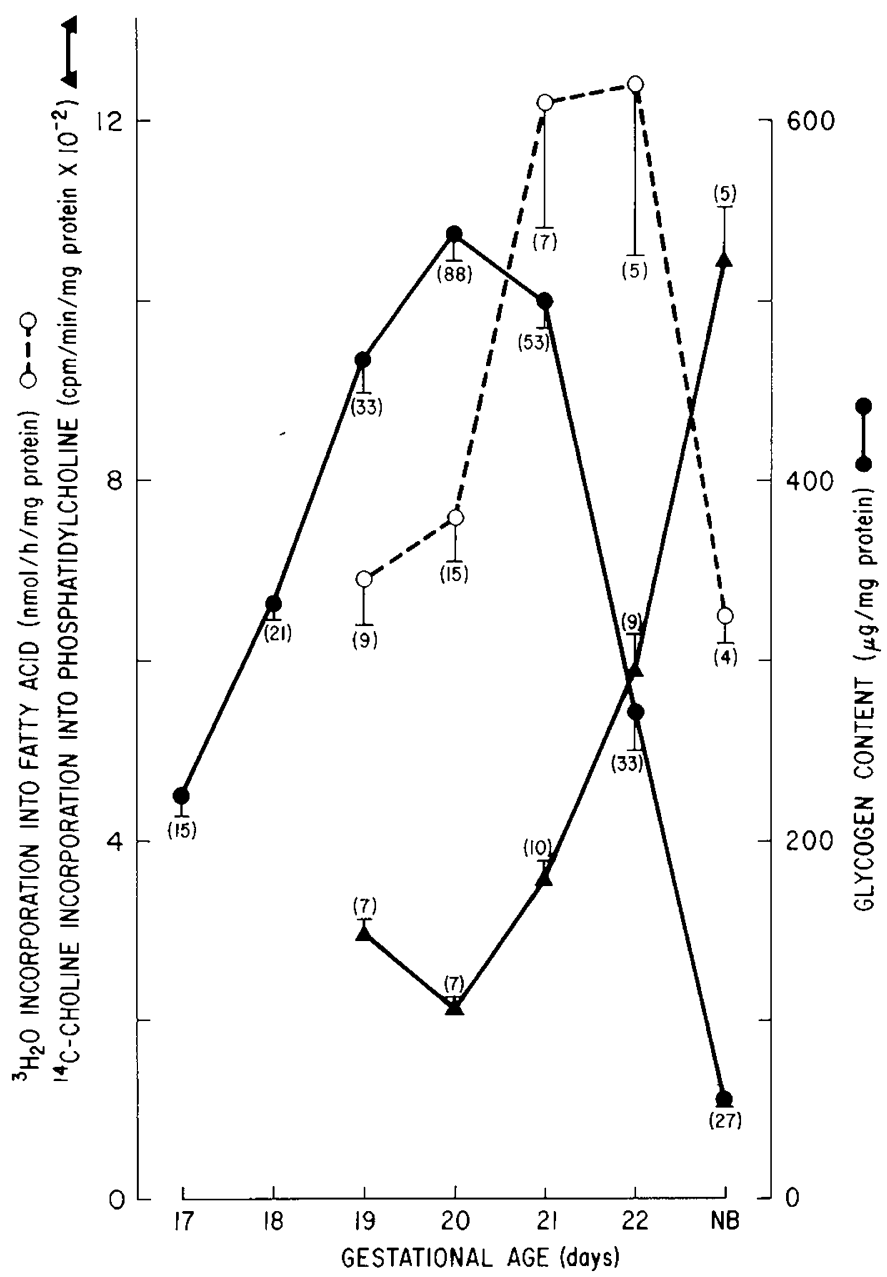

Fig. 1. Glycogen content, rate of fatty acid synthesis, and rate of choline incorporation into phosphatidylcholine in developing fetal and newborn rat lung. Data are means \pm SEM (bar) from the number of individual fetuses and newborns (glycogen content) or litters (fatty acid synthesis and choline incorporation) indicated in parentheses. Newborns $(N B)$ were 1-day-old animals. combined hormone-treated group than in the controls at this age. Although dexamethasone alone had no effect on day 20 , in combination with $\mathrm{T}_{3}$ it decreased glycogen to a greater extent than did $T_{3}$ alone. The combination also decreased lung glycogen to a greater extent than either hormone alone on day 21 . Thus the effects of dexamethasone and $T_{3}$ were antagonistic at the period in gestation when glycogen is normally increasing but additive when lung glycogen is normally decreasing.

Dexamethasone and $\mathrm{T}_{3}$ increased the rate of choline incorporation into phosphatidylcholine by 62 and $30 \%$, respectively, on day 20 (Table 3). The effect of the two hormones combined on this parameter was not examined since in a previous study using the same experimental model it was reported to be additive (8). The effects of dexamethasone and $\mathrm{T}_{3}$ on phosphatidylcholine synthesis were highly specific with respect to gestational age. Stimulation occured only on the day prior to the normal developmental surge in this parameter (Fig. 1). Thus the effect was seen on day 20 but not on days 19,21 , or 22 . It is possible that the lack of stimulation on day 19 was due to a high control value since the rate of choline incorporation into phosphatidylcholine decreased in the control groups between 19 and 20 days $(p<$ $0.005, t$ test) while in other studies it increased during this period (33-36). A decrease in the rates of choline and glycerol incorporation into phosphatidylcholine between days 18 and 19 was, however, reported in fetal rat lung (36).

The effects of the hormones on fetal weight and mortality are shown in Table 4. As in other studies in the rat (37) and rabbit (38), the fetuses from the glucocorticoid-treated animals weighed less than those from the controls. However, the differences were only statistically significant on days 18,21 , and 22 . The $T_{3}$ and combined hormone-treated groups also weighed less than the controls on days 21 and 22 . On day 21 the group treated with both hormones weighed $30 \%$ less than the controls and $24 \%$ less than those treated with either hormone alone.

There was one dead fetus among the controls on day $20(0.4 \%$ of the total) and none in the dexamethasone-treated groups (Table 4). There was increased mortality in the $\mathrm{T}_{3}$-treated groups: $1,5.7$, and $3.4 \%$ on days 20,21 , and 22 , respectively. The most striking increase in mortality, however, was in the combined hormone-treated group at 22 days when $72.3 \%$ of the fetuses were dead on delivery. In this group $2.4 \%$ were dead on day 21 but all were alive on days 17-20.

\section{DISCUSSION}

These data show that glucocorticoid and thyroid hormones have some effects in common and some opposing effects on parameters of lung maturation in the fetal rat. Both hormones accelerate the normal developmental increase in the rate of choline incorporation into phosphatidylcholine and the normal developmental decrease in lung glycogen late in gestation. However, while the glucocorticoid also accelerates the normal developmental increase in lung glycogen earlier in gestation this increase is largely prevented by the thyroid hormone. Similarly, the normal developmental increase in fatty acid synthesis is accelerated by the glucocorticoid and prevented by the thyroid

Table 1. Effect of hormones on fatty acid synthesis in fetal rat lung

\begin{tabular}{cccc}
\hline $\begin{array}{c}\text { Gestational age } \\
\text { (days) }\end{array}$ & Control & Dexamethasone & $\mathrm{T}_{3}$ \\
\cline { 2 - 4 } & \multicolumn{2}{c}{$\mathrm{nmol}^{3} \mathrm{H}_{2} \mathrm{O}$ incorporated into fatty acid methyl esters/h/mg protein } \\
\hline 19 & $6.9 \pm 0.5(9)^{*}$ & $8.4 \pm 0.6(9)$ & $5.0 \pm 0.5(3)$ \\
21 & $7.6 \pm 0.5(15)$ & $11.6 \pm 0.9(12) \dagger$ & $6.8 \pm 0.3(8)$ \\
22 & $12.2 \pm 1.4(7)$ & $12.2 \pm 1.1(4)$ & $6.5 \pm 0.4(7) \dagger$ \\
\hline
\end{tabular}

$*$ Mean \pm SEM from the number of litters in parentheses.

$\dagger$ Significantly different from control group.

$\uparrow$ Significantly different from control and dexamethasone-treated groups. 
Table 2. Effect of hormones on glycogen content of fetal rat lung

\begin{tabular}{cllcc}
\hline $\begin{array}{c}\text { Gestational age } \\
\text { (days) }\end{array}$ & \multicolumn{1}{c}{ Control } & Dexamethasone & $\mathrm{T}_{3}$ & $\mathrm{~T}_{3}+$ dexamethasone \\
\cline { 2 - 4 } 17 & $225 \pm 11(15)^{*}$ & $521 \pm 19(12) \dagger$ & $208 \pm 8(12)$ & $426 \pm 15(12) \ddagger$ \\
18 & $334 \pm 9(21)$ & $646 \pm 13(15) \dagger$ & $359 \pm 19(12)$ & $542 \pm 15(12) \ddagger$ \\
19 & $467 \pm 18(33)$ & $701 \pm 23(27) \dagger$ & $270 \pm 10(26) \dagger$ & $308 \pm 18(17) \S$ \\
20 & $537 \pm 14(88)$ & $534 \pm 14(50)$ & $285 \pm 7(47) \dagger$ & $223 \pm 17(25) \ddagger$ \\
21 & $501 \pm 14(53)$ & $371 \pm 18(34) \dagger$ & $220 \pm 8(57) \dagger$ & $152 \pm 8(37) \ddagger$ \\
22 & $272 \pm 17(33)$ & $164 \pm 6(23) \dagger$ & $93 \pm 6(29) \dagger$ & $68 \pm 8(6) \S$ \\
\hline
\end{tabular}

* Mean \pm SEM from the number of fetuses (two to three per litter) in parentheses.

$\dagger$ Significantly different from control group.

\$ Significantly different from control, dexamethasone, and $\mathrm{T}_{3}$-treated groups.

$\S$ Significantly different from control and dexamethasone-treated groups.

Table 3. Effect of hormones on rate of choline incorporation into phosphatidylcholine in fetal rat lung slices

\begin{tabular}{|c|c|c|c|}
\hline \multirow{2}{*}{$\begin{array}{c}\text { Gestational } \\
\text { age } \\
\text { (days) }\end{array}$} & Control & Dexamethasone & $\mathrm{T}_{3}$ \\
\hline & \multicolumn{3}{|c|}{$\mathrm{cpm} / \mathrm{min} / \mathrm{mg}$ protein* } \\
\hline 19 & $294 \pm 18(7)$ & $355 \pm 30(6)$ & $327 \pm 30(5)$ \\
\hline 20 & $213 \pm 11(7)$ & $345 \pm 31(8) \dagger$ & $277 \pm 18(9) \dagger$ \\
\hline 21 & $357 \pm 18(10)$ & $368 \pm 27(5)$ & $387 \pm 35(11)$ \\
\hline 22 & $588 \pm 41(9)$ & $465 \pm 58(7)$ & $510 \pm 44(7)$ \\
\hline
\end{tabular}

$*$ Mean \pm SEM from the number of litters indicated in parentheses. $\dagger$ Significantly different from the control group.

hormone. When the two hormones are administered together their effects on glycogen depletion and, as reported earlier (8), on phosphatidylcholine synthesis are additive but the stimulatory effects of the glucocorticoid on glycogen and on fatty acid synthesis are antagonized by the thyroid hormone.

Further studies are needed to elucidate the mechanism of these hormonal effects. It will be of interest to determine if the inhibitory effect of thyroid hormone on glycogen increase for instance is due to inhibition of synthesis or increased catabolism. Preliminary studies indicate that the increase in fatty acid synthesis produced by dexamethasone is accompanied by increased fattyacid synthase activity (39).

Numerous studies in several species have shown that glucocorticoid and thyroid hormones stimulate fetal surfactant production by a variety of criteria (1). In previous studies in fetal rat, rabbit, and human lung, the actions of glucocorticoid and thyroid hormones were generally reported to be additive when indices of surfactant production were measured (8-13). Betamethasone and thyroxine also had a marked additive effect on depletion of fetal heart glycogen when administered to the pregnant rabbit (40). Thyroxine, however, was reported to antagonize the stimulatory effect of cortisol on incorporation of glucose and acetate into phosphatidylcholine and phosphatidylglycerol in cultured adult rat type II pneumocytes (41), the cellular source of surfactant within the lung (1). Cortisol also increased the rates of incorporation of choline, glycerol, and palmitate into phosphatidylcholine but these effects were not antagonized by thyroxine. Since palmitate incorporation was not antagonized, the antagonistic effect of thyroxine on acetate incorporation in that study (41) could indicate an effect at the level of fatty acid synthesis. In contrast, Ballard et al. (12) reported that the stimulatory effects of $T_{3}$ and dexamethasone on rates of glucose, glycerol, and acetate incorporation into phosphatidylcholine in explants of fetal rabbit lung were additive.

In the present study we used the rate of choline incorporation into phosphatidylcholine in lung slices as an index of surfactant synthesis. Although not a specific measurement of surfactant synthesis the rate of choline incorporation into phosphatidylcholine generally shows good correlation with other indices of sur- factant production in the fetal lung $(1,14)$. Dexamethasone and $\mathrm{T}_{3}$ both increased the rate of choline incorporation into phosphatidylcholine but only on the one day prior to the normal developmental surge in this parameter. That the stimulatory effect of glucocorticoids on fetal lung phosphatidylcholine synthesis was highly specific with respect to gestational age was also reported in other in vivo and in vitro studies in the fetal rat (37, $42)$ and rabbit $(17,43)$. Glucocorticoids were reported to be most effective in preventing RDS in human infants delivered at 3034 wk gestation (5). That the effect of hormones should be confined to a relatively narrow gestational age range is not surprising if they accelerate development of a normally occurring process.

Although glucocorticoids (17-20), thyroid hormone (20), estrogen $(44,45)$, and aminophylline $(46,47)$ have previously been reported to accelerate the normal developmental decrease in lung glycogen, an acceleration of the earlier increase in lung glycogen was reported in only one previous study. Alescio and Dani (48) reported that the glycogen content of 11-day fetal mouse lung explants was increased when cultured in the presence of cortisol for $48 \mathrm{~h}$. Effects of hormones on fatty acid synthesis in developing fetal lung have not been extensively examined. Maniscalco et al. (23) reported that dexamethasone increased the rate of ${ }^{3} \mathrm{H}_{2} \mathrm{O}$ incorporation into fatty acid in fetal rabbit lung explants in vitro. In contrast, cortisol was reported to decrease the rate of de novo lung fatty acid synthesis in the same species in vivo (49). That study, however, differed from the present study and that of Maniscalco et al. (23) in at least two respects. First, incorporation of radioactivity from ${ }^{14} \mathrm{C}$-acetylCoA rather than ${ }^{3} \mathrm{H}_{2} \mathrm{O}$ was used to assess fatty acid synthesis so hormone-induced changes in the pool size of acetylCoA could account for the observed effect. Second, since the fetuses were directly injected with the hormone the inhibition might have been due to the resulting stress.

Although direct extrapolation of these findings in the fetal rat to the human infant is not warranted it is nevertheless of interest to consider whether they have any relevence to possible clinical use of a combination of glucocorticoid and thyroid hormones for prevention of RDS. Although phosphatidylcholine synthesis was increased by the hormones only on day 20 , lung maturation as determined by glycogen content was affected at all ages examined. However, while there is a temporal relationship between fetal lung glycogen depletion and increased phosphatidylcholine synthesis in several species $(1,14)$ it has not been proven that glycogen is a precursor of surfactant phospholipids (1). The relationship between the earlier developmental increase in lung glycogen and surfactant production is even more obscure. Similarly, although fatty acids are obligatory components of surfactant the relative contributions of fatty acids from de novo synthesis in the lung and from extrapulmonary sources to surfactant phospholipdis are not definitely established $(21,50)$. In addition, in the present study we measured synthesis of total lipid fatty acids rather than those in specific phospholipid molecular species. Thus, although the developmental increase in fatty acid 
Table 4. Body wt and mortality as function of gestational age and maternal hormone treatment in developing fetal and newborn rat

\begin{tabular}{|c|c|c|c|c|}
\hline $\begin{array}{l}\text { Gestational age } \\
\text { (days) }\end{array}$ & Control & Dexamethasone & $T_{3}$ & $\mathrm{~T}_{3}+$ dexamethasone \\
\hline 17 & $0.49 \pm 0.03(5)^{*}$ & $0.43 \pm 0.01$ & $0.50 \pm 0.02$ & $0.48 \pm 0.01$ \\
\hline 18 & $0.86 \pm 0.02(7)$ & $0.74 \pm 0.02(5) \dagger$ & $0.91 \pm 0.03(4)$ & $0.80 \pm 0.02(4)$ \\
\hline 19 & $1.5 \pm 0.04(16)$ & $1.4 \pm 0.05(14)$ & $1.6 \pm 0.05(11)$ & $1.5 \pm 0.11(6)$ \\
\hline 20 & $\begin{array}{c}2.3 \pm 0.05(26) \\
1 / 237 \ddagger\end{array}$ & $2.2 \pm 0.07(23)$ & $\begin{array}{c}2.4 \pm 0.06(18) \\
2 / 194\end{array}$ & $2.1 \pm 0.05(8)$ \\
\hline 21 & $3.7 \pm 0.06(21) \S$ & $3.4 \pm 0.08(12)$ & $\begin{array}{c}3.4 \pm 0.05(21) \\
16 / 282\end{array}$ & $\begin{array}{c}2.6 \pm 0.07(12) \S \\
3 / 124\end{array}$ \\
\hline 22 & $5.0 \pm 0.14(14) \S$ & $4.4 \pm 0.11(11)$ & $\begin{array}{c}4.0 \pm 0.09(12) \\
5 / 146\end{array}$ & $\begin{array}{c}3.6 \pm 0.10(6) \\
47 / 65\end{array}$ \\
\hline 1 day old & $6.2 \pm 0.18(9)$ & & & \\
\hline
\end{tabular}

\footnotetext{
per litter was $11.9 \pm 0.2$ (mean $\pm \mathrm{SE})$.

$\dagger$ Significantly different from control group.

$\ddagger$ Fetuses dead on delivery/total number of fetuses. All fetuses were alive when number is not indicated.

$\S$ Significantly different from all other groups.
}

* Means of average fetal wt $(\mathrm{g}) \pm$ SEM from the numbers of litters in parentheses. Dead fetuses were not weighed. The average number of fetuses

synthesis as well as that induced by dexamethasone coincided with the increases in phosphatidylcholine synthesis these data do not prove that such fatty acids are incorporated into surfactant. Since whole lung tissue was used in this study it is possible that not all of the effects occur in the same cell type and may not all be directly related to surfactant production. Thus, the antagonistic effects of thyroid hormone on both the developmental and glucocorticoid-induced increases in glycogen and fatty acid synthesis may have little relevence to use of these two hormones in combination for prevention of RDS. On the other hand, although a deficiency in surfactant is undoubtedly a major factor in the etiology of RDS, immaturity in other aspects of lung development may also have a bearing on it. Changes in lung elastin, for instance, were reported in infants with RDS (51) while glucocorticoids were reported to influence connective tissue in the fetal monkey (52), to increase synthesis of sulfated glycoconjugates in human fetal lung explants (53), and to increase antioxidant enzyme activities in fetal rat lung (37). Possible deleterious effects of combined hormone therapy on nonsurfactant aspects of fetal lung development should therefore be considered. It is possible that the hormones have beneficial effects at certain stages of development and deleterious effects at others. Thus the combination of hormones might be of benefit at the stage in lung development when glycogen is being depleted and surfactant synthesis is increasing but harmful at an earlier stage.

Since the litters treated with $T_{3}$ and, to a greater extent, $T_{3}$ together with dexamethasone had increased mortality as well as smaller fetuses late in gestation the issue of toxicity in combined hormone treatment ought to be considered too. In this study we used a dose of $T_{3}$ which was previously shown to optimally stimulate phosphatidylcholine synthesis in fetal rat lung at 20 days gestation (8) but did not determine if this dose was optimal at the other ages examined and did not measure hormone levels in the fetal blood. Whether a lower dose and/or a different treatment regimen would retain the beneficial effects while eliminating those that are harmful remains to be determined in the rat and other species.

\section{REFERENCES}

1. Rooney SA 1985 The surfactant system and lung phospholipid biochemistry. Am Rev Respir Dis 131:439-460

2. Liggins GC, Howie RN 1972 A controlled trial of antepartum glucocorticoid treatment for prevention of the respiratory distress syndrome in premature infants. Pediatrics 50:515-525

3. Zachman RD, Farrell PL 1982 Current recommendations for use of prenatal corticosteroids based on assessment of the risk-benefit relationship. In: Farrell PM (ed) Lung Development: Biological and Clinical Perspectives, Vol II, Neonatal Respiratory Distress. Academic Press, New York, pp 297-300

4. Mashiach S, Barkai G, Sack J, Stern E, Goldman B, Brish M, Serr DM 1978 Enhancement of fetal lung maturity by intra-amniotic administration of thyroid hormone. Am J Obstet Gynecol 130:289-293

5. Collaborative group on antenatal steroid therapy 1981 Effect of antenatal dexamethasone administration on the prevention of respiratory distress syndrome. Am J Obstet Gynecol 141:276-286

6. Ballard PL 1984 Combined hormone treatment and lung maturation. Semin Perinatol 8:283-292

7. Hitchcock KR 1979 Hormones and the lung. I. Thyroid hormones and glucocorticoids in lung development. Anat Rec 194:15-39

8. Gross I, Dynia DW, Wilson CM, Ingleson LD, Gewolb IH, Rooney SA 1984 Glucocorticoid-thyroid hormone interactions in fetal rat lung. Pediatr Res 18:191-196

9. Torday JS, Dow KE 1984 Synergistic effect of triiodothyronine and dexamethasone on male and female fetal rat lung surfactant synthesis. Dev Pharmacol Ther 7:133-139

10. Gross I, Wilson CM 1982 Fetal lung in organ culture. IV. Supra-additive hormone interactions. J Appl Physiol 52:1420-1425

11. Smith BT, Sabry K 1983 Glucocorticoid-thyroid synergism in lung maturation: A mechanism involving epithelial-mesenchymal interaction. Proc Natl Acad Sci USA 80:1951-1954

12. Ballard PL, Hovey ML, Gonzales LK 1984 Thyroid hormone stimulation of phosphatidylcholine synthesis in cultured fetal rabbit lung. J Clin Invest 74:898-905

13. Gonzales LK, Ballard PL 1984 Glucocorticoid and thyroid hormone stimulation of phosphatidylcholine synthesis in cultured human fetal lung. Pediatr Res 18:310A(abstr)

14. Rooney, SA 1983 Biochemical develoment of the lung. In: Warshaw JB (ed) The Biological Basis of Reproductive and Developmental Medicine. Elsevier Biomedical, New York, pp 239-287

15. Kikkawa Y, Kaibara M, Motoyama EK, Orzalesi MM, Cook CD 1971 Morphologic development of fetal rabbit lung and its acceleration with cortisol. Am J Pathol 64:423-442

16. Bourbon JR, Rieutort M, Engle MJ, Farrell PM 1982 Utilization of glycogen for phospholipid synthesis in fetal rat lung. Biochim Biophys Acta 712:382389

17. Rooney SA, Gobran LI, Marino PA, Maniscalco WM, Gross I 1979 Effects of betamethasone on phospholipid content, composition and biosynthesis in the fetal rabbit lung. Biochim Biophys Acta 572:64-76

18. Gilden C, Sevanian A, Tierney DF, Kaplan SA, Barrett CT 1977 Regulation of fetal lung phosphatidyl choline synthesis by cortisol: role of glycogen and glucose. Pediatr Res 11:845-848

19. Brehier A, Rooney SA 1981 Phosphatidylcholine synthesis and glycogen depletion in fetal mouse lung: developmental changes and the effects of dexamethasone. Exp Lung Res 2:273-287

20. Gross I, Wilson CM, Ingleson LD, Brehier A, Rooney SA 1980 Fetal lung in organ culture. III. Comparison of dexamethasone, thyroxine, and methylxanthines. J Appl Physiol 48:872-877

21. Maniscalco WM, Finkelstein JN, Parkhurst AB 1982 De novo fatty acid synthesis in developing rat lung. Biochim Biophys Acta 711:49-58

22. Das DK 1980 Fatty acid synthesis in fetal lung. Biochem Biophys Res Commun 92:867-875

23. Maniscalco WM, Finkelstein JN, Parkhurst AB 1985 Dexamethasone increases de novo fatty acid synthesis in fetal rabbit lung explants. Pediatr Res 19:12721277

24. Das DK, Ganguly M 1982 Mechanism of the control of pulmonary and hepatic fatty acid synthesis by the thyroid hormones. Arch Biochem Biophys 218:142-155

25. Casazza JP, Veech RL 1984 Quantitation of the rate of fatty acid synthesis. In: Story JA (ed) Lipid Research Methodology. Alan R Liss, New York, pp 231 240

26. Lowry OH, Rosebrough NJ, Farr AL, Randall RJ 1951 Protein measurement with the Folin phenol reagent. J Biol Chem 193:265-275 
27. Bligh EG, Dyer WJ 1959 A rapid method of total lipid extraction and purification. Can J Biochem Physiol 37:911-917

28. Maniscalco WM, Wilson CM, Gross I, Gobran L, Rooney SA, Warshaw JB 1978 Development of glycogen and phospholipid metabolism in fetal and newborn rat lung. Biochim Biophys Acta 530:333-346

29. Lowry OH, Passonneau JV 1972 A Flexible System of Enzymatic Analysis. Academic Press, New York, pp 189-193

30. Bourbon J, Jost A 1982 Control of glycogen metabolism in the developing fetal lung. Pediatr Res 16:50-56

31. Gewolb IH, Barrett C, Wilson CM, Warshaw JB 1982 Delay in pulmonary glycogen degradation in fetuses of streptozotocin diabetic rats. Pediatr Res 16:869-873

32. Smith BT, Tanswell AK, Minshall D, Bogues WN, Vreeken E 1982 Influence of corticosteroids on glycogen content and steroid 11 -reductase activity in lung and liver of the fetal and newborn rat. Biol Neonate 42:201-207

33. Weinhold PA 1968 Biosynthesis of phosphatidyl choline during prenatal development of the rat lung. J Lipid Res 9:262-266

34. Farrell PM 1977 Fetal lung development and the influence of glucocorticoids on pulmonary surfactant. J Steroid Biochem 8:463-470

35. Gross I, Wilson CM 1983 Fetal lung maturation: initiation and modulation. J Appl Physiol 55:1725-1732

36. Harding PGR, Chan F Casola PG, Fellows GF, Wong T, Possmayer F 1983 Subcellular distribution of the enzymes related to phospholipid synthesis in developing rat lung. Biochim Biophys Acta 750:373-382

37. Frank L, Lewis PL, Sosenko IRS 1985 Dexamethasone stimulation of fetal rat lung antioxidant enzyme activity in parallel with surfactant stimulation. Pediatrics 75:569-574

38. Possmayer F, Casola P, Chan F, Hill S, Metcalfe IL, Stewart-DeHaan PJ, Wong T, Las Heras J, Gammal EB, Harding PGR 1979 Glucocorticoid induction of pulmonary maturation in the rabbit fetus. The effect of materna injection of betamethasone on the activity of enzymes in fetal lung. Biochim Biophys Acta 574:197-211

39. Pope TS, Rooney SA 1986 Influence of glucocorticoid and thyroid hormones on enzymes of fatty acid synthesis in fetal rat lung. Fed Proc (in press)

40. Devaskar SU, Grim PF III, Devaskar UP 1985 A differential effect of thyroxine and glucocorticoids on fetal brain and heart insulin receptors. Pediatr Res 19:192-198
41. Post M, Batenburg JJ, Van Golde LMG 1980 Effects of cortisol and thyroxine on phosphatidylcholine and phosphatidylglycerol synthesis by adult rat lung alveolar type II cells in primary culture. Biochim Biophys Acta 618:308-317

42. Pysher TJ, Konrad KD, Reed GB 1977 Effects of hydrocortisone and pilocarpine on fetal rat lung explants. Lab Invest 37:588-594

43. Gross I, Ballard PL, Ballard RA, Jones CT, Wilson CM 1983 Corticosteroid stimulation of phosphatidylcholine synthesis in cultured fetal rabbit lung: evidence for de novo protein synthesis mediated by glucocorticoid receptors. Endocrinology 112:829-837

44. Khosla SS, Brehier A, Eisenfeld AJ, Ingleson LD, Parks PA, Rooney SA 1983 Influence of sex hormones on lung maturation in the fetal rabbit. Biochim Biophys Acta 750:112-126

45. Gross I, Wilson CM, Ingleson LD, Brehier A, Rooney SA 1979 The influence of hormones on the biochemical development of fetal rat lung in organ culture. I. Estrogen. Biochim Biophys Acta 575:375-383

46. Sevanian A, Gilden C, Kaplan SA, Barrett CT 1979 Enhancement of fetal lung surfactant production by aminophylline. Pediatr Res 13:1336-1340

47. Maniscalco WM, Wilson CM, Gross I 1979 Influence of aminophylline and cyclic AMP on glycogen metabolism in fetal rat lung in organ culture. Pediatr Res 13:1319-1322

48. Alescio T, Dani AM 1972 Hydrocortisone-increased glycogen deposition and its dependence on tissue interactions in mouse embryonic lung developing in vitro. J Embryol Exp Morphol 27:155-162

49. Gross I, Rooney SA, Warshaw JB 1975 The influence of cortisol on the enzymes of fatty acid synthesis in developing mammalian lung and brain. Pediatr Res 9:752-755

50. Van Golde LMG 1985 Synthesis of surfactant lipids in the adult lung. Ann Rev Physiol 47:765-774

51. Evans HE, Keller S, Mandl I 1974 Lung tissue elastin composition in newborn infants with the respiratory distress syndrome and other diseases. J Clin Invest 54:213-217

52. Beck JC, Mitzner W, Johnson JWC, Hutchins GM, Foidart JM, London WT, Palmer AE, Scott R 1981 Betamethasone and the rhesus fetus: effect on lung morphometry and connective tissue. Pediatr Res 15:235-240

53. Heifetz A, Snyder JM 1981 The effects of hydrocortisone on the biosynthesis of sulfated glycoconjugates by human fetal lung. J Biol Chem 256:49574967 